\title{
In Situ Morphology of the Aorta and Common Iliac Artery in the Fetal and Neonatal Rat
}

\author{
KAZUO MOMMA, TADAHIKO ITO, AND MASAHIKO ANDO \\ Department of Pediatric Cardiology, The Heart Institute of Japan, Tokyo Women's Medical College, \\ Tokyo, Japan
}

\begin{abstract}
In situ cross-sectional morphology of the ascending, descending, and abdominal aorta and the common iliac arteries was studied after rapid, whole-body freezing of fetal and neonatal rats. In the fetus, the ascending aorta was smaller than the descending aorta and the abdominal aorta was relatively large, continuing to the large right common iliac artery and the umbilical artery. After birth, the umbilical artery and the ductus arteriosus closed rapidly, and the size of the aorta changed within a few days. The ascending aorta became larger than the descending aorta in $\mathbf{2} \mathrm{d}$, and the abdominal aorta became smaller, especially at the infrarenal part. The right common iliac artery, which continues to the umbilical artery in fetal life, became smaller, and its diameter was the same as that of the left common iliac artery $2 \mathrm{~d}$ after birth. (Pediatr Res 33: 302-306, 1993)
\end{abstract}

Neonatal adaptation of the cardiovascular system has been studied mainly in fetal and neonatal lambs, and the physiologic aspects have been greatly clarified $(1,2)$. These include arrest of the placental circulation and closure of fetal channels, such as ductus arteriosus, ductus venosus, and foramen ovale. The arrest of the placental circulation at birth results in a sudden decrease of flow in related vessels, such as the descending and abdominal aorta, and the thoracic inferior vena cava $(1,2)$. Our early study showed decreased diameters of the thoracic descending aorta and thoracic inferior vena cava at 2 and $4 \mathrm{~d}$ after birth in the rat (3). Recently, Langille et al. (4) studied the thoracic descending aorta and abdominal subrenal aorta in fetal and neonatal lambs and reported a decreased diameter of the subrenal aorta a few weeks after birth. However, they studied only these two parts of the aorta, and postnatal changes in the rest of the aorta remain obscure. In this report, postnatal changes of the entire aorta were observed in the rat with use of a recently developed method of studying in situ morphology of the cardiovascular system of the rat without postmortem changes $(3,5-7)$.

\section{MATERIALS AND METHODS}

Animals. Twenty virgin Wistar rats (pregnancy period $21.5 \mathrm{~d}$ ) were mated overnight from 1700 to $0900 \mathrm{~h}$, and the presence of sperm in vaginal smears fixed day 0 of pregnancy. These rats were fed commercial solid food and water. The average litter size was 13. Treatment of the rats conformed to the guiding principles of the American Physiological Society.

Freezing, cutting, and photographing. Fetal and neonatal vas-

Received August 4, 1992; accepted October 30, 1992.

Correspondence: Kazuo Momma, M.D., The Heart Institute of Japan, Tokyo Women's Medical College, Kawadacho 8-1, Shinjuku-ku, Tokyo, 162, Japan.

Supported by a grant-in-aid from the Ministry of Education, Science, and Culture of Japan and by the Japan Research Promotion Society for Cardiovascular Diseases. cular morphology was studied using the rapid whole-body freezing technique, as previously reported $(3,5-7)$. For fetal studies, six pregnant rats were killed on the $21 \mathrm{st} d$ by cervical dislocation and frozen immediately in liquid nitrogen, followed by removal of the frozen fetuses. For the newborn rat study, 14 mother rats nursed newborns for $1,2,3$, or $4 \mathrm{~d}$, at which time the newborns were frozen in dry ice-acetone. For the study of neonates immediately after birth, fetuses were delivered by cesarean section from two rats on the $21 \mathrm{st} \mathrm{d}$, the umbilical cord was cut bluntly, and the fetuses were frozen immediately in dry ice-acetone. To avoid possible effects of arrest of the umbilical circulation, some fetuses were delivered by cesarean section from two other rats on the 21 st $\mathrm{d}$ and were frozen immediately in dry ice-acetone with intact umbilical cord and placenta. These were defined as neonates with intact umbilical circulation.

The frozen thorax and abdomen were trimmed and sectioned on a freezing microtome (Komatsu Solidate Co., Tokyo, Japan) in a transverse plane. Cross-sectioned surfaces were photographed with a binocular stereoscopic microscope (Wild M 400 Photomakroscope, Wild Heerbrugg Ltd., Heerbrugg, Switzerland), using color film (Reala, Fuji Film Co., Tokyo, Japan) serially at every $500-\mu \mathrm{m}$ width. Numbered section paper $(1 \times 1$ $\mathrm{mm}$ ) was photographed for scale, and the pictures were printed on paper.

Measurement. The inner diameter of the ascending aorta was measured between the aortic valve and the aortic arch. The aortic isthmus was measured between the origin of the left subclavian artery and the junction of the ductus arteriosus. The thoracic descending aorta was measured at the ventricular level. The abdominal infrarenal aorta was measured at a point distal to the origin of the renal arteries. The right and the left common iliac arteries were measured immediately distal to their bifurcation. The shorter diameter was assumed to be the arterial diameter if the artery was cut obliquely and was ovoid.

Statistical analysis. Morphometric data are presented as mean \pm SEM. Comparisons between the fetus and other groups were subsequently submitted to $t$ test at a confidence level of $95 \%$.

\section{RESULTS}

The ascending aorta was slightly smaller than the main pulmonary artery and the thoracic descending aorta in the fetus (Fig. 1A). After birth, an immediate increase of about $15 \%$ occurred in the vascular diameter of both the ascending and descending aorta in neonates with intact umbilical circulation as well as in those whose umbilical cord was bluntly cut (Fig. 2, Table 1). The diameters of the ascending aorta and aortic isthmus were smallest $1 \mathrm{~d}$ after birth and became larger $2 \mathrm{~d}$ after birth (Fig. 2, Table 1). The diameter of the thoracic descending aorta decreased within the first $2 \mathrm{~d}$ after birth (Figs. 2 and $3 B$, Table 1).

Neonatal decrease in aortic diameter was more prominent in the more distal aorta. The diameter of the abdominal suprarenal 


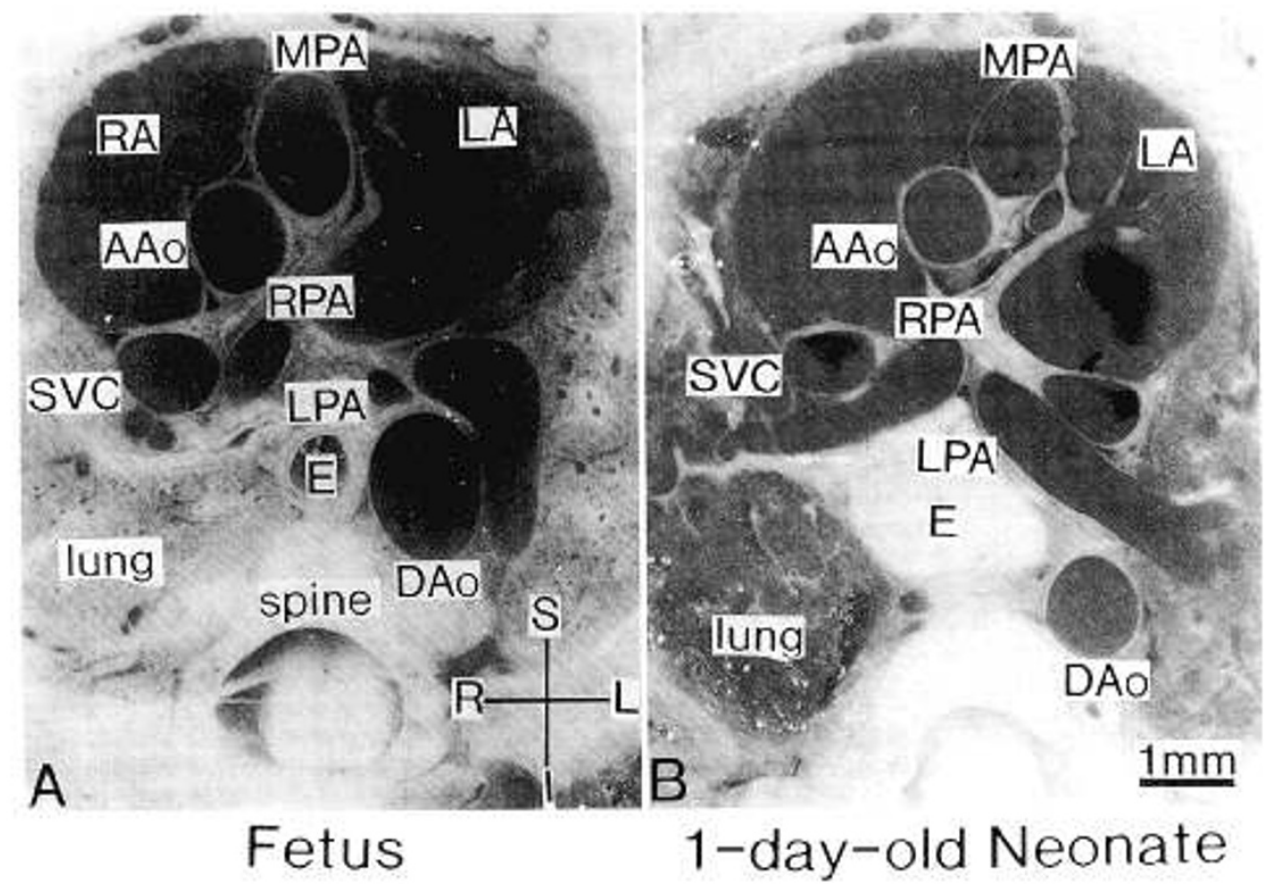

Fig. 1. Transverse section of the upper thorax in the fetus $(A)$ and 1-d-old neonate $(B)$. The scale is the same in $A$ and $B$ in Figures 1, and 3-6. Note smaller descending aorta in the neonate compared with the fetus. $A A O$, ascending aorta, $D A O$, descending aorta; $E$, esophagus; $I$, inferior; $L$, left; $L A$, left atrium; $L P A$, left pulmonary artery; $M P A$, main pulmonary artery; $R$, right; $R A$, right atrium; $R P A$, right pulmonary artery; $S$, superior; and $S V C$, superior vena cava.

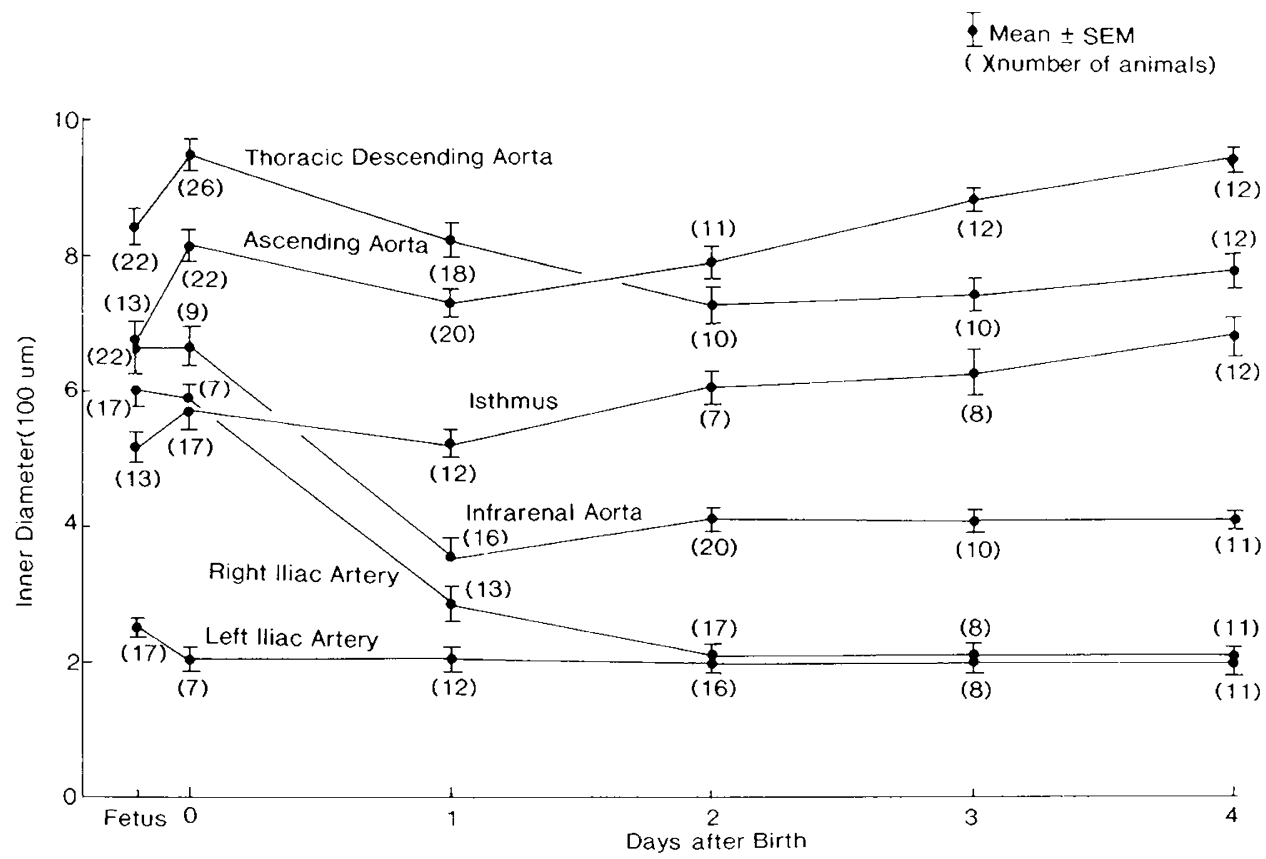

Fig. 2. Inner diameters of the aorta and iliac arteries of the fetal and neonatal rats. Mean \pm SEM. Numbers in parentheses are number of animals studied.

aorta became smaller $2 \mathrm{~d}$ after birth (Fig. 4B). This postnatal decrease in aortic size was more prominent at the infrarenal aorta (Figs. 2 and 5B, Table 1). The diameter of the infrarenal aorta became half that of the fetal size at $1 \mathrm{~d}$ after birth (Fig. 2). The right common iliac artery continued to the umbilical artery and was very large in the fetus (Fig. 6A). The left common iliac artery did not continue to the umbilical artery and was small in the fetal rat. Right common iliac artery diameter decreased rapidly after birth and was the same size as the left common iliac artery $2 \mathrm{~d}$ after birth (Figs. 2 and $6 B$, Table 1).

\section{DISCUSSION}

Growth of the great vessels in the fetus has become a topic of interest and has been studied both echocardiographically and at autopsy $(8,9)$. Postnatal changes in these great vessels are interesting because of arrest of the placental circulation and closure of fetal channels, such as the ductus arteriosus, ductus venosus, and foramen ovale. However, very little attention has been paid to postnatal changes in the size of the aorta and common iliac arteries $(1,2)$. 
Table 1. Inner diameters of the aorta and common iliac arteries of fetal and neonatal rats*

\begin{tabular}{llllll}
\hline & Fetus & Neonate and IUC & Neonate $-0 \mathrm{~min}$ & $1 \mathrm{~d}$ & $2 \mathrm{~d}$ \\
\hline $\begin{array}{l}\text { Body wt }(\mathrm{g}) \\
\text { Inner diameters of the aorta }(10 \mu \mathrm{m})\end{array}$ & $5.0 \pm 0.1(20)$ & $4.8 \pm 0.1(11)$ & $5.1 \pm 0.1(23)$ & $5.2 \pm 0.1(12) \dagger$ & $7.6 \pm 0.2(13) \dagger$ \\
$\quad$ Ascending Ao & $68 \pm 3(13)$ & $77 \pm 2(11) \dagger$ & $81 \pm 2(22) \dagger$ & $74 \pm 2(20) \dagger$ & $79 \pm 2(11) \dagger$ \\
Isthmus & $51 \pm 3(13)$ & $60 \pm 2(6) \dagger$ & $57 \pm 3(17) \dagger$ & $53 \pm 2(12)$ & $61 \pm 2(7) \dagger$ \\
Thoracic descending Ao & $84 \pm 2(2)$ & $96 \pm 2(11) \dagger$ & $96 \pm 2(26) \dagger$ & $82 \pm 2(18) \dagger$ & $73 \pm 2(10) \ddagger$ \\
Abdominal infrarenal Ao & $67 \pm 3(22)$ & $66 \pm 4(9)$ & $67 \pm 3(9)$ & $36 \pm 2(16) \dagger$ & $41 \pm 2(20) \dagger$ \\
Right common iliac A & $60 \pm 1(17)$ & $57 \pm 2(6)$ & $57 \pm 4(7)$ & $29 \pm 4(13) \dagger$ & $21 \pm 1(17) \dagger$ \\
Left common iliac A & $23 \pm 1(17)$ & $20 \pm 1(6)$ & $20 \pm 2(7)$ & $21 \pm 1(12)$ & $21 \pm 1(16)$ \\
\hline
\end{tabular}

$*$ Values are mean \pm SEM with number of animals in parentheses. A, artery; Ao, aorta; IUC, intact umbilical circulation.

$\dagger p<0.05$ vs fetus.

$\ddagger p<0.05$ vs 0 -minute neonate.
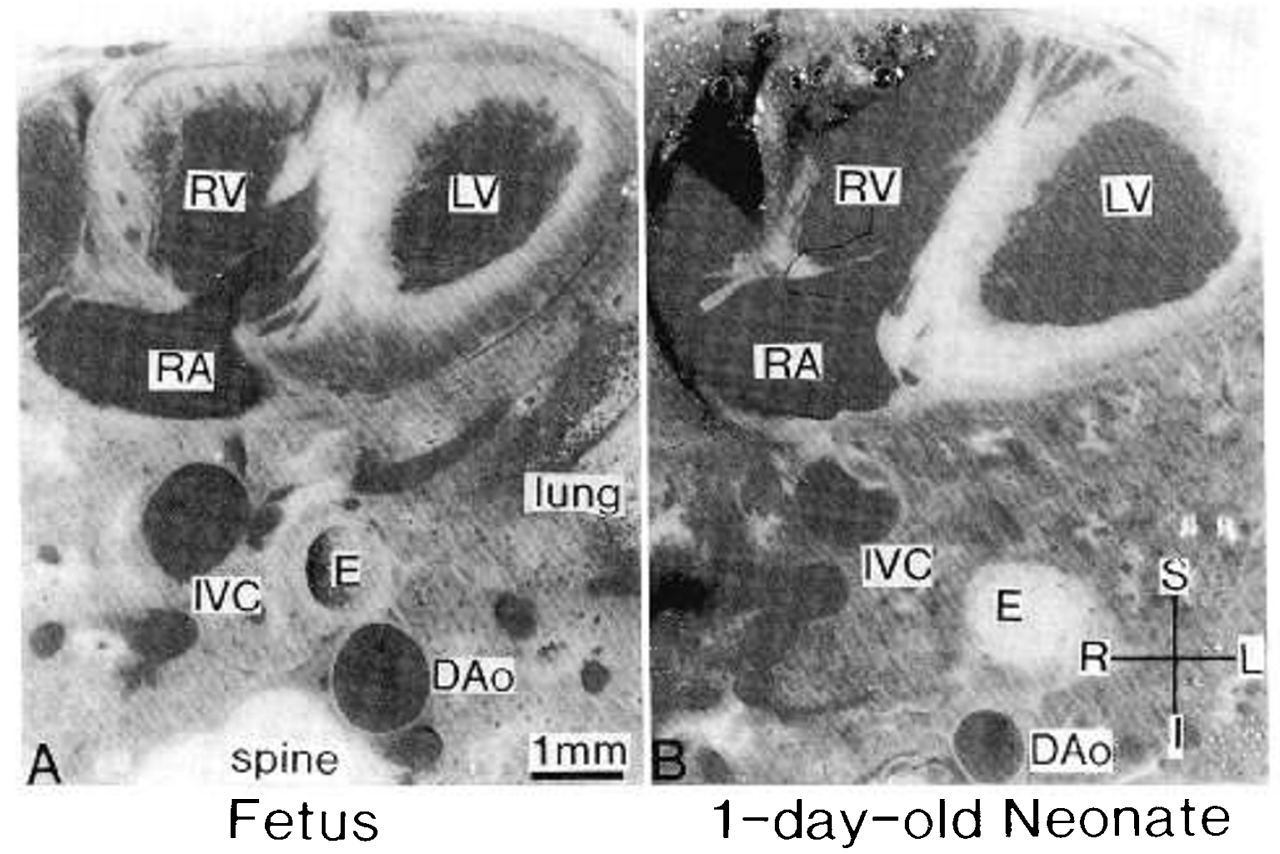

Fig. 3. Transverse section of the middle thorax in the fetus $(A)$ and 1-d-old neonate $(B)$. Note smaller descending aorta in the neonate compared with the fetus. $I V C$, inferior vena cava; $R V$, right ventricle; and $L V$, left ventricle. Other abbreviations are the same as in Figure 1.

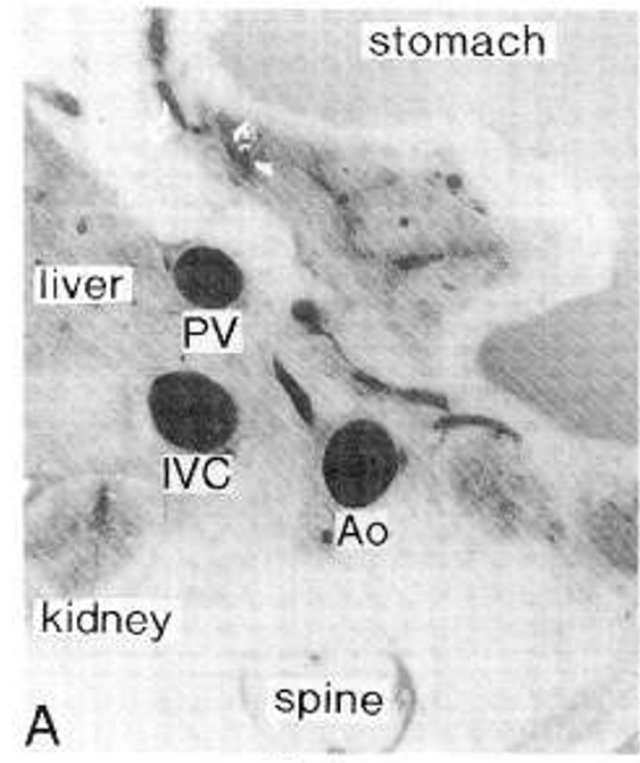

Fetus

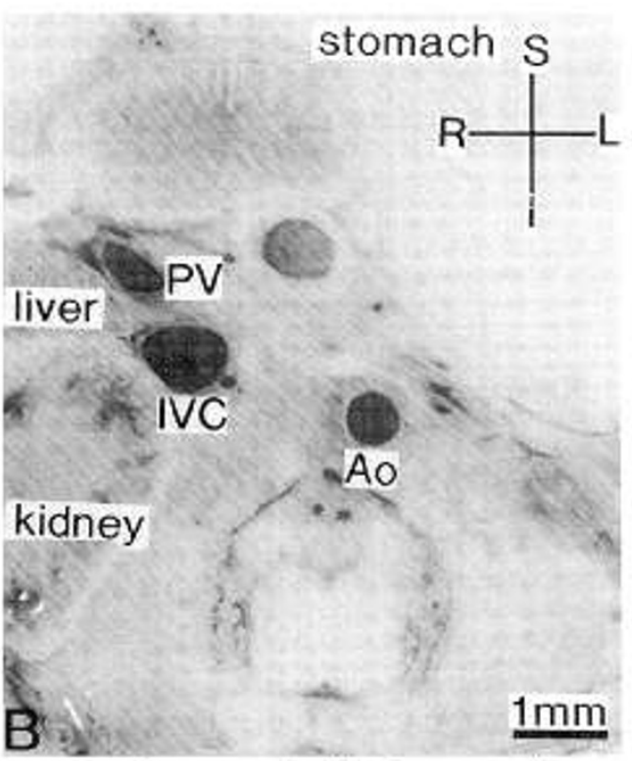

2-day-old Neonate

Fig. 4. Transverse section of the upper abdomen in the fetus $(A)$ and 2-d-old neonate $(B)$. Note smaller descending aorta in the neonate compared with the fetus. $A O$, aorta; and $P V$, portal vein. Other abbreviations are the same as in Figures 1-3. 


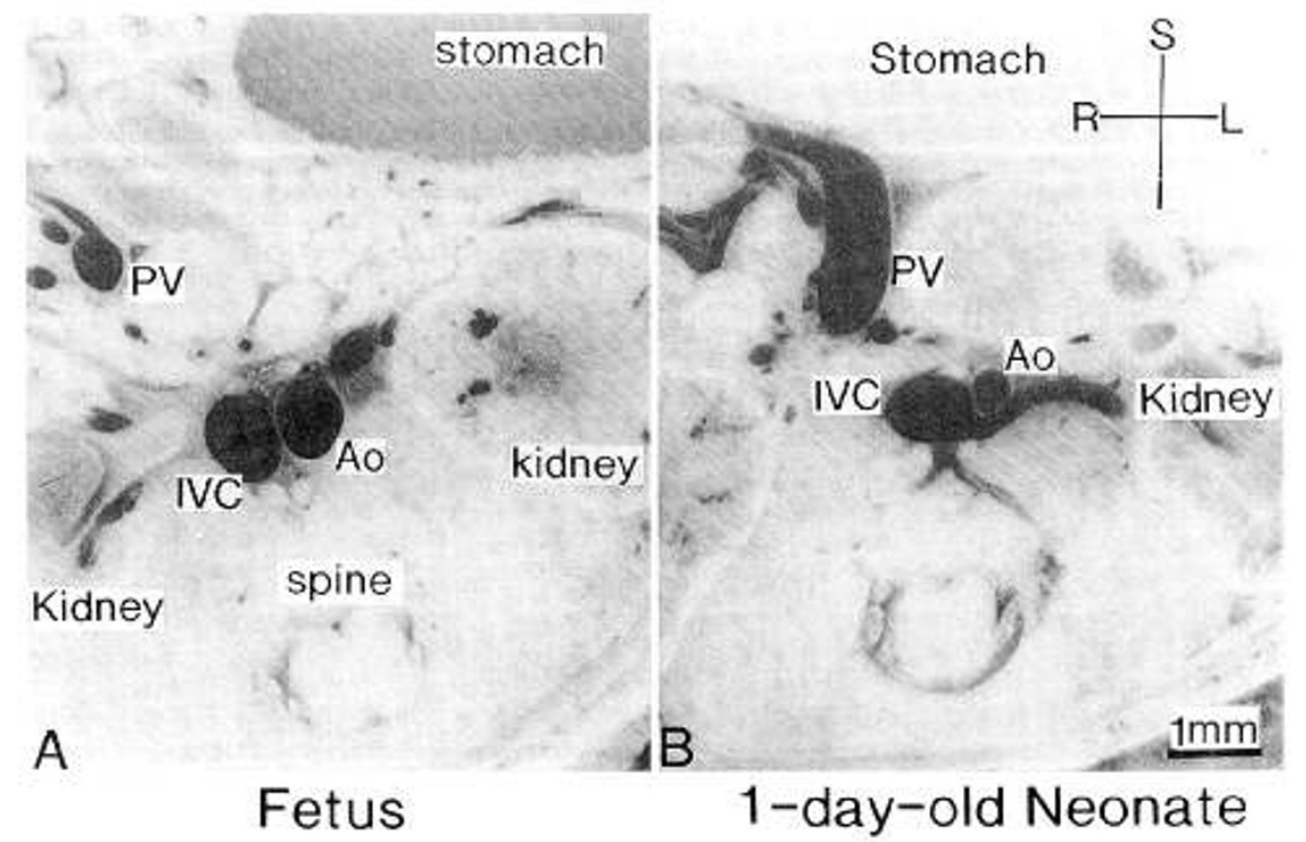

Fig. 5. Transverse section of the middle abdomen in the fetus $(A)$ and 1-d-old neonate $(B)$. Note smaller descending aorta in the neonate compared with the fetus. Other abbreviations are the same as in Figures 1-4.

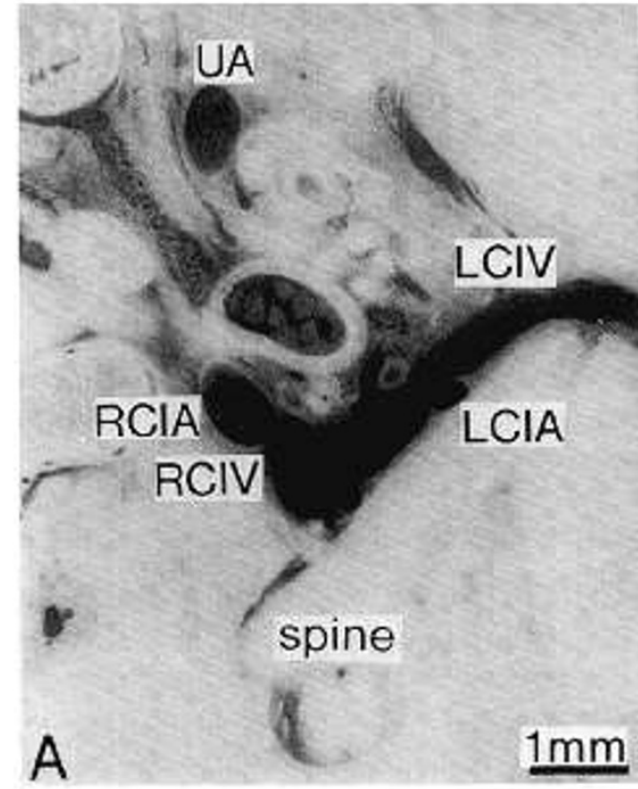

Fetus

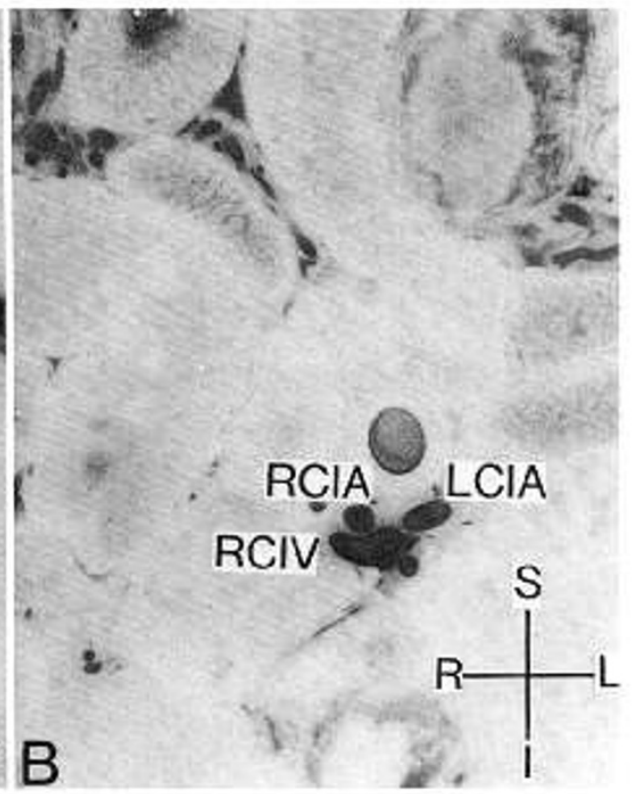

2-day-old Neonate

Fig. 6. Transverse section of the lower abdomen in the fetus $(A)$ and 2-d-old neonate $(B)$. Note large right common iliac artery and large umbilical artery in the neonate compared with the fetus. $L C I A$, left common iliac artery, $L C I V$, left common iliac vein; $R C I A$, right common iliac artery; $R C I V$, right common iliac vein; and $U A$, umbilical artery. Other abbreviations are the same as in Figures $1-5$.

Our present study demonstrated rapid postnatal change of the aortic diameter in the rat. Presumably, these changes are adaptations in accord with the physiologic principle of minimal work (11). According to this principle, the vascular system adapts to blood flow by changing diameter to a necessary minimum (11), and it has been deduced theoretically that vascular diameter is proportionate to the cube root of the flow (11). Although no absolute blood flow data of the aorta are available in fetal and neonatal rats, a rapid increase in left ventricular output in the neonate has been reported. The reported increase compared with fetal values was $30 \%(2)$ and $100 \%$ (12) in the lamb, and $20-$ $30 \%$ (13) and $40 \%$ (14) in the human infant. Blood flow through the isthmus was $50 \mathrm{~mL} / \mathrm{kg} / \mathrm{min}$ in the fetal lamb (2), and it is calculated to be more than $100 \mathrm{~mL} / \mathrm{kg} / \mathrm{min}$ in the neonate, assuming that it is more than half of the cardiac output.

Another remarkable observation in this study was a rapid decrease in the diameters of the neonatal abdominal aorta and the right common iliac artery that carried blood to the placenta in fetal life. In contrast to the double umbilical arteries in the human fetus, the rat fetus has a single umbilical artery that is an extension of the right common iliac artery (10). Therefore, our study demonstrated a remarkable postnatal change in the right in comparison to the left common iliac artery in the rat. Blood flow in the thoracic descending aorta was about $345 \mathrm{~mL} / \mathrm{kg} /$ $\mathrm{min}$, and blood flow to the placenta was about $200 \mathrm{~mL} / \mathrm{kg} / \mathrm{min}$ in the fetal lamb (2). After birth, umbilical circulation ceased, 
and cardiac output and blood flow to the ascending aorta was about $200 \mathrm{~mL} / \mathrm{kg} / \mathrm{min}(2)$. Although blood flow in the descending aorta was not known, it was presumed to be much less than blood flow in the ascending aorta. Therefore, blood flow in the descending aorta was much less than fetal blood flow in the lamb. Flow through the descending aorta and the common iliac artery in the fetal and neonatal rat is not known. The diameter of the infrarenal aorta decreased from 0.67 to $0.36 \mathrm{~mm} 1 \mathrm{~d}$ after birth in this study. This change corresponds to decreased blood flow to $16 \%$ of the fetal flow, according to the theoretical formula.

Changes were rapid and completed in a day or two after birth in the rat. Although these changes in aortic size have not been studied in human neonates, they are presumably slower in the human because of slower growth and closure of fetal channels. For example, the ductus arteriosus closes about $15 \mathrm{~h}$ or more after birth in human neonates (2) and closes about $1 \mathrm{~h}$ after birth in neonatal rats (7). Body weight doubles within 3 mo after birth in human infants and within $5 \mathrm{~d}$ of age in neonatal rats (15).

Acknowledgment. The authors thank Barbara Levene and Dr. L. M. Linde, Professor of Pediatric Cardiology, University of Southern California School of Medicine, for editorial assistance.

\section{REFERENCES}

1. Dawes GS 1968 Foetal and Neonatal Physiology. Year Book Medical Publishers, Chicago, pp 79-89

2. Rudolph AM 1974 Congenital Diseases of the Heart. Year Book Medical Publishers, Chicago, pp 1-28
3. Momma K, Takao A, Ito R, Nishikawa T 1987 In situ morphology of the heart and great vessels in fetal and newborn rats. Pediatr Res 22:573-580

4. Langille BL, Brownlee RD, Adamson SL 1990 Perinatal aortic growth in lambs: relation to blood flow changes at birth. Am J Physiol 259:H1247$\mathrm{H} 1253$

5. Momma K, Takao A 1990 Right ventricular concentric hypertrophy and left ventricular dilatation by ductal constriction in fetal rats. Circ Res 64:11371146

6. Momma K, Ando M, Takao A 1990 Fetal cardiac morphology of tetralogy of Fallot with absent pulmonary valve in the rat. Circulation 82:1343-1351

7. Momma K, Konishi H, Hagiwara H 1985 Characteristic morphology of the constricted fetal ductus arteriosus following maternal administration of indomethacin. Pediatr Res 19:493-500

8. Ursell PC, Byrne JM, Fears TR, Strobino BA, Gersony WM 1991 Growth of the great vessels in the normal human fetus and in the fetus with cardiac defects. Circulation 84:2028-2033

9. Angelini A, Allan LD, Anderson RH, Crawford DC, Chita SK, Ho SY 1988 Measurements of the dimensions of the aortic and pulmonary pathway in the human fetus: a correlative echocardiographic and morphometric study. Br Heart J 60:221-226

10. Hebel R, Stromberg MW 1986 Anatomy and Embryology of the Laboratory Rat. BioMed Verlag, Wörthsee, Germany pp 248

11. Murray CD 1926 The principle of minimum work. I. Proc Natl Acad Sci USA $12: 204-214$

12. Kirkpatrick SE, Covell JW, Friedman WF 1973 A new technique for the continuous assessment of fetal and neonatal cardiac performance. Am J Obstet Gynecol 116:963-972

13. Wladimiroff JW, Vosters R, McGhie JS 1982 Normal cardiac ventricular geometry and function during the last trimester of pregnancy and early neonatal period. Br J Obstet Gynecol 89:839-844

14. Agata Y, Hiraishi S, Oguchi K, Misawa H, Horiguchi Y, Fujino N, Yashiro K, Shimada N 1991 Changes in left ventricular output from fetal to early neonatal life. J Pediatr 119:441-445

15. Anversa P, Olivetti G, Long AV 1980 Morphologic study of early postnatal development in the left and right ventricular myocardium of the rat. I. Hypertrophy, hyperplasia, and binucleation of myocytes. Circ Res 46:495502

\section{Announcement}

\section{Call for Abstracts}

Abstracts are now being accepted for the Fifth Congress of the European Society of Pediatric Neurology, to be held in Strasbourg, France, November 11-13, 1993. For further information, contact Joseph Haddad, M.D., Service de Pediatrie, University Hospital of Strasbourg, Hopital de Hautepierre, F 67098 Strasbourg Cedex, France; tel: 3388289000 , ext. 2470; fax: 3388271061. 